\title{
MODEL ATMOSPHERES AND RADIATIVE TRANSFER IN CHEMICALLY PECULIAR STARS: \\ INTERPRETATIONAL SIGNIFICANCE OF NON-LTE
}

\author{
I. Hubený \\ Astronomical Institute \\ Crechoslovak Academy of Sciences \\ 25165 Ondřjov, Czechoslovakia
}

\section{INTRODUCTION}

One of the main problems in studying chemically peculiar (CP) stars is the question of the extent to which the conceptual framework of the contemporary spectroscopic diagnostics is reliable. As the first step, it should be clarified whether the traditional assumption of local thermodynamic equilibrium (LTE) provides an adequate approximation to reality, or whether a more general non-LTE approach should be employed.

The period of rapid development of computational methods and extensive calculations of NLTE model atmospheres is now past its culmination point. The importance of a relevant NLTE description is viewed as unquestionable for hot stars ( $B$ and earlier). Consequently, the hot stars attract the attention of most "NLTE theoreticians," while considerably less attention is being devoted to later types (late B and A). Moreover, the abundance anomalies found in the CP stars are no longer expected to be a spurious result of an inadequate (LTE) analysis (Cowley 1981). It is not commonly accepted that other phenomena such as diff usion, magnetic field, inhomogeneous abundance distribution, etc., are quite essential ingredients of the general atmospheric pattern of $\mathrm{CP}$ stars. Therefore, a large part of the astronomers investigating the CP stars now consider the NLTE effects to be rather minor perturbations of the LTE predictions.

Is LTE indeed a satisfactory description of the CP stellar atmospheres, and if so, why? For what purposes? On the other hand, is an NLTE approach really necessary, and if so, why? For what diagnostic purposes? For what spectral types and/or spectral features? These are the most urgent questions which are of interest to both observers and theoreticians.

It is the aim of the present paper to discuss the questions raised above. We stress at the very outset that we shall only consider the so-called classical stellar atmospheres, i. e. those characterized by the assumptions of plane parallel horizontally homogeneous stratification, hydrostatic and radiative equilibrium, but not generally local thermodynamic equilibrium (LTE). Primary attention will be devoted to examining the changes in atmospheric structure and emergent spectrum that arise due to relaxing the assumption of LTE. Excellent reviews of the classical model stellar atmospheres, as well as those that relax one or more of the above assumptions can be found in Mihalas (1978) and in the series of NASA-CNRS monographs "Non-thermal Phenomena in Stellar Atmospheres" (Underhill and Doazan, 1982; Wolff, 1983; Thomas, 1983; and references therein). Further, we shall not consider more involved treatments of the classical NLTE radiative transfer as, for instance, departures from complete frequency redistribution in spectral lines (for recent reviews of this topic, see Linsky, 1985 and Hubený, 1985).

We shall primarily concentrate on the methodological problems, leaving aside the more technical aspects of the theory (for details, refer to the excellent textbook of Mihalas, 1978) as well as the discussion of the results of actual diagnostic studies (for a review see Wolff, 1983; and some other papers of this conference). An illuminating discussion of some general aspects 
of spectroscopic diagnostics of CP stars may be found in interesting papers by Cowley (1980, 1981), Praderie (1982), and Cowley and Adelman (1983).

\section{PRELIMINARY CONSIDERATIONS}

\subsection{LTE versus NLTE}

Generally speaking, NLTE is a state of radiation and matter characterized by any kind of departure from the LTE. However, this term is of ten used in a limited sense. In the usual astrophysical context, the difference between LTE and NLTE consists only in the manner of calculating the atomic level populations.

Briefly, under the assumptions of LTE, the occupation numbers of bound and free states of atoms (level populations) at a specified point in the atmosphere are functions of only two thermodynamic variables - electron temperature and density - and are given by the well known Saha-Boltzmann equations. This is no longer generally true under the assumption of NLTE; instead, atomic level populations have to be determined by solving the statistical equilibrium equations which express the balance of all microscopic processes populating and depopulating the various atomic states. No other departures from LTE distribution functions are allowed in this restricted NLTE approach; this led some authors to propose more appropriate names, such as the statistical equilibrium (Wolff 1983) or kinetic equilibrium (Athay 1972) approach. However, we retain the name NLTE throughout the present paper.

It is customary to express NLTE level populations in terms of the LTE populations that correspond to the same local values of temperature and electron density as

$$
n_{i}=b_{i} n_{i}^{*}
$$

where $n_{i}$ is the actual (NLTE) population, $n_{i}$ the corresponding LTE population, and $b_{i}$ is the so-called departure coefficient ( $b$-factor) of a given level $i$.

The solution of the statistical equilibrium equations approaches the LTE distribution (b $\rightarrow 1$ ) if either the collisional rates dominate over the radiative rates, or the radiation field approaches the equilibrium-Planckian-distribution. Taking into account the typical atmospheric conditions, the physical state of a stellar atmosphere may be characterized by a continuous transition from deep layers, where LTE prevails, to more superficial layers, where NLTE is more and more important.

Finally, it should be stressed that the (astrophysical) (LTE) already represents a nonequilibrium state of the radiation matter complex. The approximation of LTE allows for the most pronounced non-equilibrium property of stellar atmospheres: the radiation escapes the atmosphere (we see the star !) and, therefore, cannot generally possess a Planckian distribution. The actual radiation field is then determined by solving the radiative transfer equation; the opacity and the source function are, however, assumed independent of the radiation field. The LTE modeling also allows for an indirect influence of radiation on the thermodynamic parameters via the equation of radiative equilibrium. The only feature which is neglected by the LTE approach is the influence of radiation on the atomic level populations (and thus on the opacity and source function). Anyway, the LTE approach accounts for many essential features of stellar atmospheres; this explains its relative success in atmospheric modelling (see below).

\subsection{Coupling versus Decoupling}

To take into account all the microscopic processes populating and depopulating the atomic levels, thereby allowing for the coupling between all the populations and state parameters, is clearly beyond the state of the art. It is thus necessary to disregard, or crudely simplify, a vast 
majority of individual microscopic processes, believing that those which were neglected are indeed negligible.

Thus, while the coupling between processes (state parameters) is q quite essential NLTE feature, the idea of decoupling is the actual gist of any application. The usual decoupling, which is virtually always made, is a two-step calculation of predicted spectral features (e.g. line profiles): i) calculation of the model atmosphere, adopting LTE, etc., and ii) having been given the model atmosphere, the radiative transfer and the statistical equilibrium equations are solved for an individual atom or ion, taking into account as much detail as feasible (and reasonable). The assumption underiying this approach is that the detailed behavior of the level populations and the radiation field in the lines and continua of this atom (ion) do not affect the overall atmospheric structure significantly.

Notice that a decoupling is inevitably involved in the second step as well. Only a limited number of atomic levels and transitions between them is accounted for explicitly; the remaining ones are treated either approximately (e.g. in LTE) or are ignored completely. Consequently, careful test calculations are, in principle, always required to verify that nothing important has been overlooked.

However, there is one problem that makes the safe applicability of the two-step procedure doubtful. This problem is usually referred to as line blanketing, and represents a task of accounting for a large number of spectral lines which influence both the atmospheric structure and the emergent spectrum. Since every single spectral line is, at least in principle, affected by departures from LTE, and since we are interested in the cumulative effect of many lines, the very nature of the decoupling of the atmospheric structure and the detailed line transfer is violated.

On the other hand, in judging the efficiency and suitability of a modelling approach, one should be aware of an intimate link between the degree of sophistication of the theoretical calculations, on the one hand, and the intended interpretational goal on the other. Obviously, less accurate observations may be interpreted by means of simpler models; various spectral features in various stars of ten require different kinds of models.

Specifically, the following diagnostic scheme, which is parallel to the two-step atmospheric modelling, is commonly accepted: Step (i) - model atmospheres - is viewed as a procedure that yields the continuum flux, while step (ii) - detailed transfer solutions - is viewed as a procedure needed to predict detailed line profiles. Once again, line blanketing complicates the matter significantly, yet some features of the two-step procedure are found here as well. Schematically, less accurate treatments of numerous lines are used to compute the atmospheric structure and predicted continua, while more accurate treatments are used to compute detailed synthetic spectra.

There is another source of difficulties that may hamper or even inhibit accurate modelling. This is the necessity of knowing precisely an enormous amount of atomic data, such as oscillator strengths, photo-ionization cross-sections, collisional rates, line broadening parameters, etc. A particularly useful review of this problem in the context of CP stars is given by Cowley and Adelman (1983).

We have tried to summarize the above considerations in Figure 1. Further on, we shall discuss the three basic steps of modelling-model atmospheres, detailed line transfer, and line blanketing - in turn. Before doing this, we advance a simplified picture which will prove useful in the subsequent analysis.

\subsection{Simplest NLTE Situations}

Although the nature of the NLTE effects is generally quite complex, it may of ten be visualized as the result of superposition of or competition among some elementary phenomena. However, this idea must be used with great caution. Although useful, such a simplistic view may be quite misleading in certain cases.

Let us restrict ourselves to the case of a semi-infinite atmosphere with no incident 


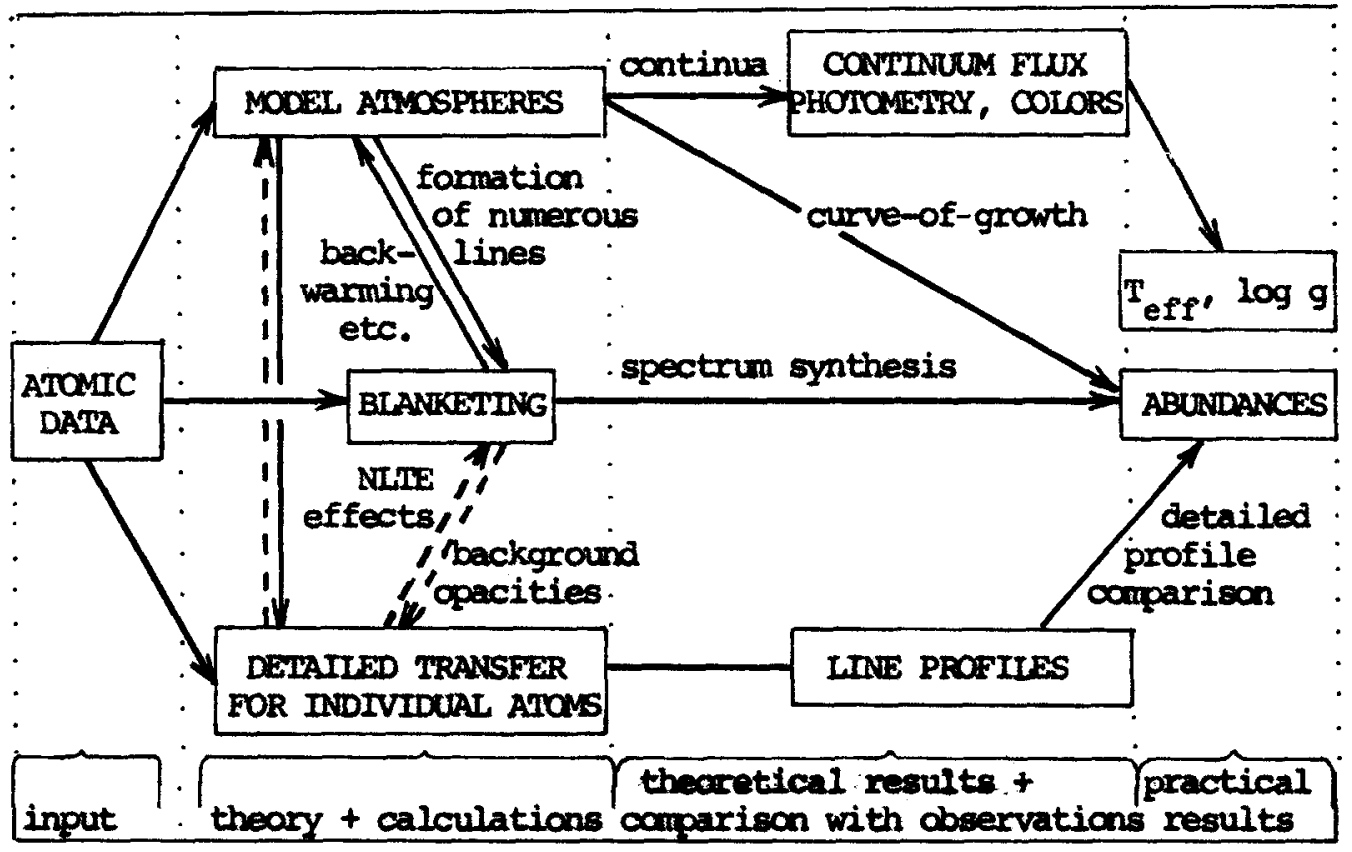

Figure 1. A Schematic diagram suggesting the logical connections between the different steps of atmospheric modelling and spectroscopic diagnostics discussed in the text. The full lines represent the standard procedures that have already been (at least partly) elaborated; the broken lines represent the procedures to be worked out.

radiation at the surface. There are now in principle two kinds of NLTE effects: 1) those which arise due to the presence of an outer boundary (a lack of photons that have escaped through the boundary affects the balance of photo-excitations and de-excitations or photoionizations and recombinations), irrespective of the presence of gradients of the thermodynamic parameters (temperature, density); the gradients may still modify the magnitude of these effects; and ii) effects that arise due to the presence of gradients. Three different cases can thus be distinguished which are displayed schematically ion Figure 2.

Case A, a typical NLTE effect, represents the behavior of a strong resonance line (or a strong ground state continuum, e.g. the hydrogen Lyman continuum). Cases B and C are typical of continua. In both these cases, the mean intensity $\mathrm{J}_{\nu}$ decreases towards the surface, where it roughly equals $(1 / 2) \mathrm{S}_{\nu}\left(\tau_{\nu}=1\right) \simeq(1 / 2) \mathrm{B}_{\nu}, \mathrm{S}_{\nu}$ being the source function and $\mathrm{B}_{\nu}$ the Planck function. Consider a bound state $i$. The photo-ionization rate is proportional to $\int \mathrm{J}_{\nu} \ldots \mathrm{d} \nu$, while the recombination rate is proportional to $\int \mathrm{B}_{v} \ldots \mathrm{d} \nu$. Now if the statistical equilibrium from level $i$ is dominated by radiative ionizations and recombinations (which occurs at depths where the lines are saturated while the continua are not) then $b_{i}>1$ if $J_{\nu}<B_{\nu}$ (case $\mathrm{B}$ ), and $\mathrm{b}_{\mathrm{i}}<1$ if $\mathrm{J}_{\nu}>\mathrm{B}_{\nu}$ ( case $\mathrm{C}$ ).

Case $\mathrm{C}$ represents the characteristic behavior of the continua formed at wavelengths for 
which the gradient of the Planck function is sufficiently steep (roughly, shortward of the maximum of the Planck function for a temperature equal to the effective temperature). Actually for $A$ and late $B$ stars, the type $C$ behavior is typical of the continua formed in the Balmer continuum.

Obviously, our scheme is neither exhaustive nor complete. Yet, while other similar schemes (see, e.g., Mihalas 1978; Freire and Praderie 1974) are devised primarily to understand the behavior of NLTE line profiles, our scheme is meant to be helpful in understanding the global NLTE multilevel, multitransition radiative transfer in lines and continua of complex atoms or ions.

\section{MODEL ATMOSPHERES}

\subsection{LTE Models}

Let us consider separately model atmospheres of normal and "peculiar" stars, the distinction between both groups being roughly given by the adopted chemical composition, or, sometimes, by including magnetic fields.

3.1.1. Normal Stars. By far the most extensive grid of LTE model atmospheres available at present is Kurucz's (1979). Line blanketing of about one million atomic lines is accounted for statistically by means of the opacity distribution functions. This grid, supplemented by the corresponding theoretical ubvy and UBV photometric indices, calculated by Relyea and Kurucz (1978) and Buser and Kurucz (1978), respectively, provides a widely used norm to which observed stellar spectra and colors are compared.

Concerning the spectroscopic diagnostics of A and late B stars, Kurucz himself showed that there is an excellent agreement between his model predictions and the spectroscopic observations of Vega. Afterwards, many investigators employed Kurucz's models and compared the predicted and observed flux distributions. Systematic studies of a sample of stars have been carried out, e.g. by Underhill et al. (1979), Böhm-Vitense (1981), and Malagnini et al. $(1982,1983)$. Generally, these authors have demonstrated that Kurucz's models reproduce the observed visual distribution fairly well, while the UV flux is reproduced somewhat worse, but still satisfactorily. However, the present observational uncertainties in the ultraviolet do not allow definite conclusions concerning model deficiencies to be drawn.

Kurucz's grid meets most of the practical requirements, hence only a few other LTE model calculations of normal stars have recently been carried out. We mention the study of Dreiling and Bell (1980), who have calculated a detailed line blanketed model atmosphere for Vega, and compared the theoretical predictions with the observations in the wavelength range $1250<\lambda<10800 \mathrm{~A}$. They again found satisf actory agreement, except in the far UV region ( $\lambda$ $<1500 \mathrm{~A}$ ) where, however, the observational uncertainties are the largest.

3.1.2. LTE model atmospheres for peculiar stars. The published model atmospheres can be divided into two categories which mimic the two most pronounced characteristics of the peculiar stars, namely i) models with enhanced metal abundances, with otherwise the same assumptions as adopted for modelling the atmospheres of normal stars; and ii) models that account for the effects of magnetic fields.

The models of the first category basically aim at describing quantitatively the phenomena expected on simple theoretical grounds: an enhanced metal opacity in the ultraviolet decreases the UV flux and increases the visible flux via increased backwarming in the deep atmospheric layers. Strom and Strom (1969) and Peterson (1970) suggested that continua of common elements, e.g. Si, may supply such an enhanced opacity. (However, subsequent NLTE calculations showed that continua alone are insufficient - see below). A more realistic study has been carried out by Leckrone et al. (1974) who were able to demonstrate convincingly that 
1) BOUNDARY EFFECTS
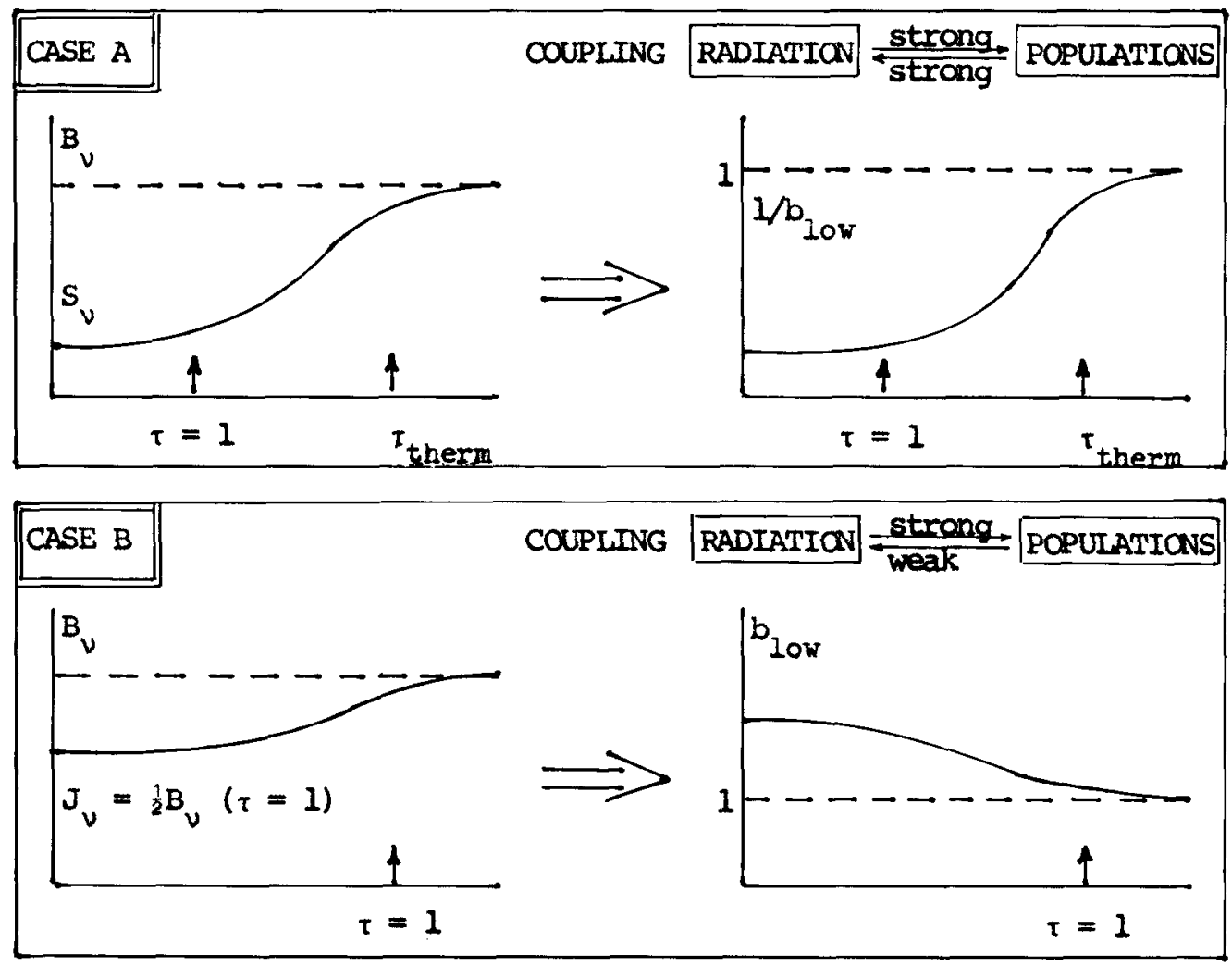

2) GRADIENT EFFECTS

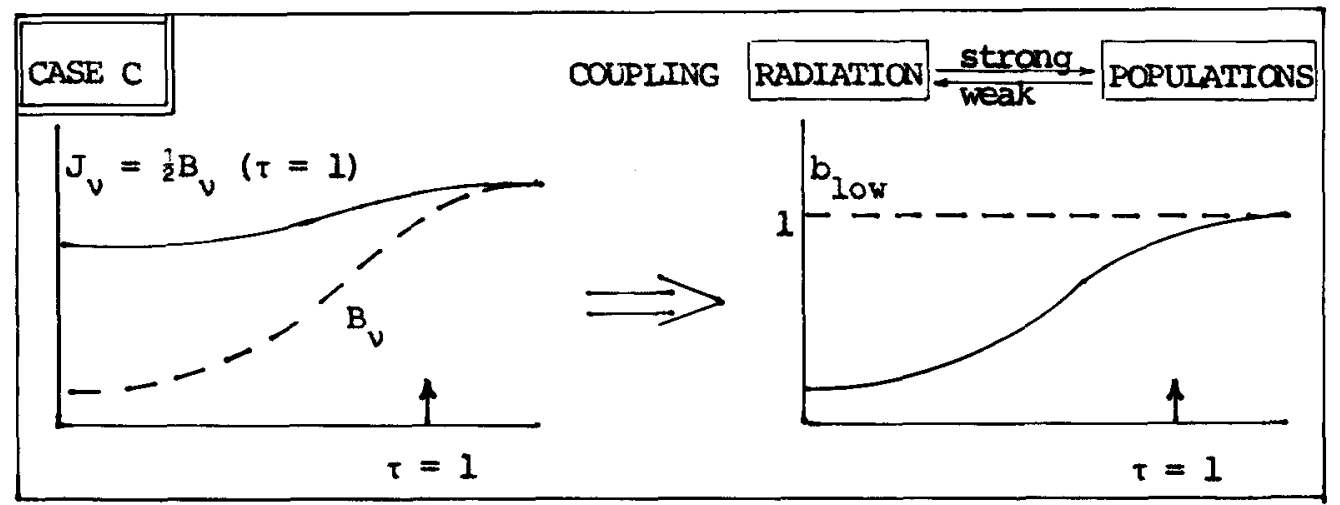

Figure 2. A highly schematic diagram illustrating the simplest NLTE situations. Left-hand graphs - see text; right-hand graphs - the corresponding b-factors for the lower level of a given transition.

an enhanced line opacity model simulates a hotter atmosphere in the visible and a cooler atmosphere is the UV, similar to what is actually observed. 
Subsequently, a similar but more extensive study was undertaken by Muthsam and coworkers. Line blanketing of about

$\mathrm{x} 10^{5}$ spectral lines has been accounted for by means of the opacity sampling procedure suggested by Peytremann (1974); representative abundances for CP stars have been adopted. Magnetic fields were not considered in the equation of hydrostatic equilibrium, but their effects on the formation of spectral lines (by Zeeman broadening) have been mimicked by introducing a pseudo-microturbulent velocity (Muthsam 1979). Besides calculating a grid of models (Muthsam 1978), they presented a comparison of the theoretical predictions with observations, both for a sample of Ap stars (Stepien and Muthsam 1980) and for individual stars (Muthsam and Stepien 1980; Stepien and Muthsam 1981; Muthsam and Cowley 1984). Some individual features present in the Ap-star spectra have also been considered by the spectral synthesis technique (Muthsam and Weiss 1978; Maitzen and Muthsam 1980).

There is a number of further studies that either employ Kurucz's (1979) models or calculate their own model atmospheres using a methodology similar to Kurucz's, and compare the theoretical predictions with observations for various types of CP stars [e.g. Kurucz and Furenlid 1979; Bell and Dreiling 1981 - Sirius; Lane and Lester 1984 - Am stars; Baschek et al. $1984-\lambda$ Boo stars, etc. ]. Attention has also been devoted to the search, by means of the LTE spectral synthesis, for extra opacity sources in the atmospheres of Ap stars (autoionization lines - Jamar et al. 1978; Jamar 1980; Artru et al. 1981; Artru and Lanz - this conference).

Generally, the LTE models have been proven to provide a good explanation of the many observed features of $\mathrm{CP}$ stars, thereby demonstrating that the classical methodology of computing line blanketed LTE model stellar atmospheres is indeed an efficient approach to modelling CP steilar atmospheres, which is worth pursuing further. On the other hand, many individual features, particularly in the ultraviolet but also in the visible, remain unexplained.

Finally, we shall briefly mention the models of the second category. An earlier paper by Stepien (1978) concluded that the effects of magnetic fields should be rather small. However, subsequent, more complete calculations by Madej (1983) indicate that the magnetic pressure can significantly influence the atmospheric structure and the emergent spectrum of an Ap star. Yet, these models do not consider metal line blanketing.

Carpenter (1985) has recently taken a major step forward by calculating LTE model atmospheres that take into account both the magnetic pressure and the line blanketing effects. He demonstrated that the structure changes (via alteration of the gas pressure distribution due to magnetic forces) in combination with enhanced blanketing (via Zeeman broadening of lines) cause the emergent spectrum to vary with the viewing inclination and to differ from the nonmagnetic case, and concluded that these model atmospheres of magnetic Ap stars are consistent with the observations.

\subsection{NLTE Model Atmospheres}

3.2.1 Why calculate NLTE models? As discussed in the preceding section, LTE model atmospheres have been shown to provide an excellent basis for the interpretation of the observed spectra of normal stars, and a somewhat poorer but still satisfactory basis for CP stars. Moreover, numerous studies seem to indicate that the discrepancies between observations and theory are likely to be caused by observational errors and/or other physical mechanisms (inhomogeneous abundances, magnetic fields, etc.) rather than by departures from LTE. The natural question then arises whether to calculate NLTE model atmospheres for A and late B type stars.

In our opinion, the answer is affirmative due to the following reasons: i) The NLTE description of the interaction between the radiation and matter is principally superior to the LTE one. If for nothing else, the NLTE models can serve to justify the applicability of the LTE models. ii) NLTE is an inherent part of radiative transfer physics. While phenomena like inhomogeneous abundances or magnetic fields may or need not be actually present, the NLTE 
effects are unequivocally, to a higher or lesser degree, present always. Hence, NLTE phenomena should be understood well, otherwise a misinterpretation of the observations in terms of spurious phenomena may easily occur.

From a more practical point of view, NLTE models are necessary iii) in order to decide for which spectral features the departures from LTE are substantial; iv) to treat the uppermost layers of an atmosphere and related spectral features (cores of the strongest lines, $X$-radiation, etc.). Here, and NLTE treatment is obligatory, yet further refinements of the theory are still required.

\subsubsection{Overview of NLTE model calculations $\left(T_{\text {eff }} \leq \underline{15000 \mathrm{~K})}\right.$. In spite of the great conceptual}

importance of NLTE model calculations, only a few studies have been undertaken so far. The first NLTE, pure hydrogen model atmosphere in this temperature range ( $\mathrm{T}_{\text {eff }}=12500 \mathrm{~K}$ ) was calculated by Auer and Mihalas (1970). They demonstrate that this model exhibits a behavior similar to that found for higher temperatures as, for instance, an increase of the electron temperature in the uppermost layers (due to the indirect effect of the Balmer lines on the continuum energy balance.

Analogous calculations for cooler atmospheres were carried out by Frandsen (1974) and Kudritzki (1973). Frandsen calculated the NLTE models for $T_{\text {eff }}=10000 \mathrm{~K}, \log (\mathrm{g})=4$ (and also 2, and 1); Kudritzki considered hydrogen-helium atmospheres and calculated NLTE models for $\mathrm{T}_{\text {eff }}=10000 \mathrm{~K}, \log (\mathrm{g})=1$, for various ratios $\mathrm{N}(\mathrm{He}) / \mathrm{N}(\mathrm{H})$. Finally, Borsenberger and Gros (1978) have extended Mihalas's (1972) grid of NLTE model atmospheres towards cooler stars. They presented a grid of NLTE model atmospheres for $10000 \leq T_{\text {eff }} \leq 15000 \mathrm{~K}, \log (\mathrm{g})=4$. The atmospheres were assumed to be composed of hydrogen, helium [with $\mathrm{N}(\mathrm{He}) / \mathrm{N}(\mathrm{H})=0.1$ ] and an average of $\mathrm{C}, \mathrm{N}, \mathrm{O}$, with $\mathrm{N}(\mathrm{CNO}) /$ $\mathrm{N}(\mathrm{H})=10^{-3}$.

In all of the above models, departures from LTE were allowed for the first five levels of hydrogen; the hydrogen $\mathrm{Ha}, \mathrm{H} \beta, \mathrm{Pa}, \mathrm{P} \beta$, and $\mathrm{Ba}$ lines (except for Kudritzki's models) were taken into account explicitly in the statistical equilibrium equations. The Lyman lines were taken to be in detailed balance. The most important conclusions drawn from these studies are: i) the energy distribution in the continua is little affected by departures from LTE, except at very low gravities and in the (yet unobserved) Lyman continuum; and ii) significant departures from LTE occur in the cores of the Balmer and Paschen lines, the wings being described rather well by LTE.

On the other hand, it was recognized during the seventies that the NLTE effects in hydrogen are not the most important NLTE effects to be expected for A and late B stars. For instance Snijders $(1977 \mathrm{a}, \mathrm{b})$, who did not actually calculate model atmospheres, but used Kurucz's models and recalculated the emergent radiation, demonstrated that the NLTE effects in neutral carbon (actually $C$ type effects in our terminology) affect the emergent far UV radiation considerably. Consequently, it became clear that the NLTE modelling of the $A$ and late $B$ atmospheres should represent more than a straightforward extension of the methodology used for $B$ and $O$ stars.

More realistic model atmospheres of early A type stars $\left(9400 \leq \mathrm{T}_{\text {eff }} \leq 9700 \mathrm{~K}, \log (\mathrm{g})=\right.$ 4), allowing for departures from LTE in $\mathrm{H}_{1} \mathrm{H}^{-}, \mathrm{C} \mathrm{I}, \mathrm{Si}$ I, N I, S I and $\mathrm{Mg}$ II, were calculated by Hubeny (1981). The effects of line wing opacities of the La to $L \delta$ lines were included in the calculations, allowing for the departures from complete frequency redistribution (Hubeny 1980). In agreement with the previous NLTE studies, the effects of departures from LTE have been found to be negligible in the visible and near UV continua $(\lambda>1700 \AA)$ while, in contrast, NLTE effects were found to be important for the far UV region $(\lambda<1500 \AA)$. In particular, for the region $1100<\lambda<1200 \AA$, the LTE flux is too low by a factor of as much as 50! Good agreement has been found between the predicted flux for the NLTE model with 
$T_{\text {eff }}=9660, \log (g)=4$, and the flux observed for Vega in which the UV and visible, and even in the region $1100<\lambda 1200 \AA$, which was previously a point of controversy (see Praderie et al 1975; Snijders 1977b; Praderie 1981).

All the above studies ignore the effects of metal line blanketing. The only attempt to mimic these effects in NLTE model atmospheres is due to Borsenberger and Jamar (1980) who studied the influence of an enhanced UV opacity on atmospheric structure. They found the behavior to be analogous to that described by Leckrone et al. (1974) for LTE models. However, their approach is questionable since they considered departures from LTE only in hydrogen and not in the most important UV absorbers (C, Si, etc.).

\subsection{Discussion}

The main interpretational conclusions following from the above-mentioned studies are summarized in Table 1. Although very schematic, this table illustrates several important methodological considerations outlined earlier.

Table 1: Spectroscopic Diagnostics of Continua

\begin{tabular}{|c|c|c|}
\hline Region & $\begin{array}{l}\text { Diagnostic } \\
\text { method }\end{array}$ & Main problems \\
\hline IR continue & LTE & circumstellar matter (?) \\
\hline $\begin{array}{l}\text { Vis continua } \\
\text {-colors } \\
\text {-special photometry } \\
\text { (Geneva, Maitzen) }\end{array}$ & $\begin{array}{l}\text { LTE } \\
\text { LTE? }\end{array}$ & $\begin{array}{l}\text { (blanketing) } \\
\text { blanketing,magnetic fields } \\
\text { NLTE ionization shifts }\end{array}$ \\
\hline - near UV continua & LTE? & $\begin{array}{l}\text { blanketing, magnetic fields, } \\
\text { atomic parameters, NLTE } \\
\text { ionization shifts }\end{array}$ \\
\hline far continua & NLTE & $\begin{array}{l}\text { blanketing, atomic parameters, } \\
\text { details of NLTE treatment }\end{array}$ \\
\hline EUV, $\mathrm{X}$ continua & $\begin{array}{l}\text { more exact } \\
\text { physics }\end{array}$ & practically not tackled yet \\
\hline
\end{tabular}

From the practical point of view, the diagnostic method indeed depends on the spectral region and/or the precision of observations. Roughly speaking, LTE model atmospheres appear to be a satisfactory diagnostic tool for continua of $\mathrm{A}$ and late $\mathrm{B}$ stars. On the other hand, more detailed observations and/or the far UV region require an NLTE treatment.

From the methodological point of view, NLTE model atmospheres have already proven their usefulness. However, it should be kept in mind that their true value still lies in the philosophy of the problem rather than in practical applications. As an example, consider the far UV region in early A stars - see Fig. 3. The LTE and NLTE predictions for $T_{\text {eff }}=9600 \mathrm{~K}$ 
are quite different; the NLTE prediction yields an almost perfect agreement with observations for Vega (Hubený 1981). Moreover, the NLTE predictions indicate that the violet wing of La is very sensitive to the effective temperature. Consequentiy, this region might seem to be an excellent indicator of $\mathrm{T}_{\text {eff }}$. However, this is not quite so, since various uncertainties and approximations in the relevant atomic data and the treatment of the far UV opacity sources yield large differences in the calculated emergent flux. The corresponding scatter in the calculated departure coefficients for the $\mathrm{C} I$ ground state is given in Fig. 4. (for details refer to Hubený 1981).

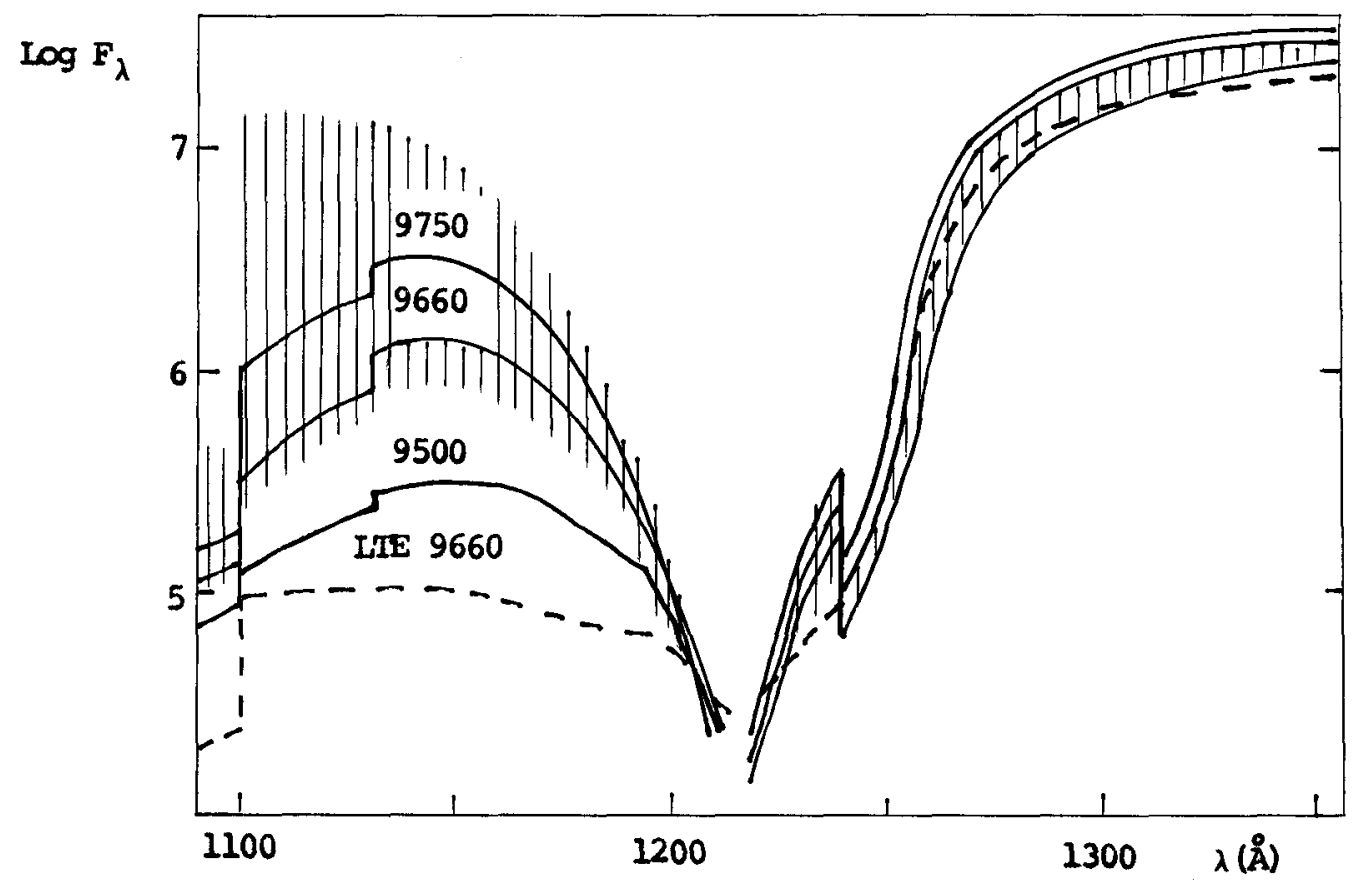

Figure 3. Predicted far UV flux for NLTE (full lines) and LTE (broken lines) model atmospheres. The curves are labeled with the corresponding value of $T_{\text {eff }}$. The shaded area represents the range of the emergent flux for $\mathrm{T}_{\text {eff }}=9660$ calculated under various approximations in the treatment of the far UV opacity sources (after Hubený 1981).

In summing up, NLTE models, although certainly important, are not yet capable of providing a reliable and accurate norm to which the observations can be compared in detail. The crucial problem to be solved is the inclusion of metal line blanketing in the NLTE model atmospheres. We shall return to this question in Section 5.

\section{DETAILED TRANSFER SOLUTIONS FOR INDIVIDUAL ATOMS}

\subsection{Overview}

NLTE studies of the individual atoms/ions are more common than those of model atmospheres. However, not all such studies have been motivated solely by reasons explained in 


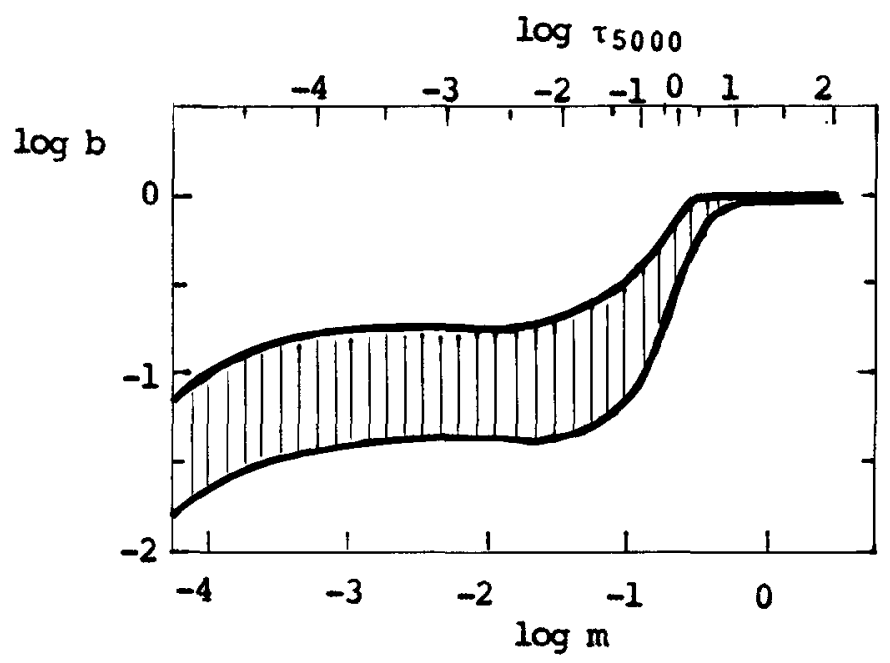

Figure 4. The corresponding range of the departure coefficient for the $\mathrm{C} I$ ground state calculated under various approximations in the treatment of the far UV opacity sources (after Hubený 1981).

Sect. 2.2. In fact, many NLTE studies have been carried out to examine numerically the parameter-f ree model of diffusion of chemical elements in CP stars (Michaud 1970, 1981, this conference; for computational details refer to Borsenberger 1979).

The calculations performed so far for A and late B stars are summarized in Table 2. For completeness, the solutions for atoms/ions, which were calculated self-consistently with the global atmospheric structure (and hence, strictly speaking, do not belong to the present category of studies), are also included.

From the computational point of view, the majority of studies use a "direct" approach: several lowest atomic levels are treated explicitly; higher levels are either disregarded or treated approximately. An entirely different approach has been adopted by Alecian and Michaud (1981). To cope with the complicated energy level structure of $\mathrm{Mn} \cdot \mathrm{III}$, they did not consider the individual energy levels separately; instead, they lumped several levels with similar energies together, assuming that all levels within each group have equal b-factors. Such an approach represents a promising way of examining NLTE effects in other "complicated" atoms, such as the iron group elements.

The main purpose of Table 2 is to extract the general features of the individual solutions. An inspection of Table 2 reveals two important conclusions which were, in fact, already suggested earlier (cf. Praderie 1976; Snijders 1977a), namely: i) For ions with the ionization limit in the Lyman continuum and with no low-lying levels collisionally coupled with the ground state, there are small continuum effects; line effects dominate the behavior of level populations; ii) For ions with the ionization limit in the Balmer continuum, or in the Lyman continuum but with at least one collisionally coupled low-lying level, the ionization energy of which lies in the Balmer continuum, there are important C-type continuum effects (see Sect. 2.3).

In the latter case, the departure coefficients of all levels considered explicitly exhibit the behavior displayed schematically in Figs 2(C) and 4. Higher up in the atmosphere, the line effects start to influence the populations. Despite the complex nature of these phenomena, two types of behavior may again be distinguished: 
a) for abundant atoms ( $\mathrm{C}, \mathrm{Mg})$ - the A-type effects completely dominate the formation of resonance lines, while the subordinate lines are still more or less affected by the $C$-type continuum effects;

b) for less abundant atoms ( $\mathrm{Be}, \mathrm{B}, \mathrm{Ca}, \mathrm{Sr}, \mathrm{Ba}$ ) - the resonance lines are now weaker; the result of the competition between the A-type effects for resonance lines and C-type for continua is very sensitive to the adopted abundance as well as to the actual values of the relevant atomic parameters. The $\mathrm{C}$-type continuum effects of ten dominate the formation of the subordinate lines.

\subsection{Ionization Shif ts}

The latter conclusion is particularly important from the point of view of abundance determinations and line blanketing studies. It shows that the C-type effects may dominate the formation of many observable lines which are thus formed as if the abundance of a given ion were lower than the corresponding LTE abundance - the so-called ionization shift. However, this does not necessarily mean that the total abundance of the ion is actually lower than the LTE abundance. For instance, if the population of the ground state significantly exceeds the total population of the excited states, and if the A-type effects dominate the formation of the resonance lines, then $\mathrm{N}_{\text {ion }}<\mathrm{N}_{\text {ion }}^{*}$ at depths where continua are transparent but resonance lines are still saturated (pure C-type), and $\mathrm{N}_{\text {ion }}>\mathrm{N}_{\text {ion }}^{*}$ at depths where $\mathrm{b}_{1}>1$ (i.e. where the A-type effects dominate over the C-type).

The ionization shift is an important NLTE effect because it can systematically affect the formation even of weak lines. Its relative simplicity indicates the possibility, to be employed more fully in future calculations, of considering NLTE effects for a large number of lines without performing heavy calculations. Specifically, were we able to estimate quantitatively the ionization shifts for individual ions, we could calculate synthetic spectra and NLTE blanketed model atmospheres by methods analogous to those employed in the LTE calculations.

On the other hand, the present state of the matter does not allow such quantitative estimates to be made reliably. We are able, at least, to summarize the favorable conditions for the occurrence of the ionization shift (cf. Snijders 1977a):

1) The next ion is the dominant ionization degree of a given atom.

2) The next ion is predominantly in the ground state.

3) The given ion is predominantly in a few low-lying states.

4) One has the C-type continuum effect at least for one low-lying level.

Briefly, conditions 1 and 2 are necessary to obtain an actual ionization shift. If they are not satisfied, an increase of the ionization increases the ground-state population of the next ion substantially, thereby also increasing the recombination rate and thus the population of the lower ion. If condition 3 is not satisfied, the C-type effects may be overwhelmed by coupling (radiative and collisional) with other lines and continua; condition 4 is obvious.

The following ions have already been proved to fulfill all these requirements for the conditions found in the atmospheres of A and late B stars: C I, N I, Si I, S I, Mg II, Ca II, Sr II, $\mathrm{Ba}$ II. Taking into account the atomic level structure and the relevant ionization potentials, we may reasonably expect ionization shif ts for the following ions: $\mathrm{P} \mathrm{I}, \mathrm{Cl} \mathrm{I,} \mathrm{Mn} \mathrm{I,} \mathrm{Fe} \mathrm{I,} \mathrm{Co} \mathrm{I,}$ $\mathrm{Ni}$ I, Cu I, Zn I, Y II, Zr II, Hg I, etc., and, importantly, for once ionized rare earths.

It is interesting to note that Cowley (1980) suggested, on purely observational grounds that the LTE ionization equilibrium for the rare earth elements is probably violated. Our estimates show that the NLTE ionization shifts act in the right sense towards removing the observational discrepancies, but, clearly, this question deserves further attention. 
TABLE 2: NLTE Transfer Solutions for Individual Atoms

\begin{tabular}{|c|c|c|c|c|}
\hline Atom/ion & Reference & Purpose & $\begin{array}{l}\text { Continuum } \\
\text { effects }\end{array}$ & Line effects \\
\hline $\begin{array}{l}\text { Be II } \\
\text { Be I,II }\end{array}$ & $\begin{array}{l}\text { Boesgaard et al. (1982) } \\
\text { Borsenberger et al (1984) }\end{array}$ & $\begin{array}{l}\text { LWA } \\
\text { D }\end{array}$ & - & type A res.lines \\
\hline B II & Praderie et al. (1977) & $\mathrm{LA}(\mathrm{N})$ & Type C & $b_{2}>b_{1}$ \\
\hline B II,III & Borsenberger et a1. (1979) & DNWL & Type C & (weak res. line) \\
\hline C I & $\begin{array}{l}\text { Snijders }(1977 a, b) \\
\text { *Hubený }(1981)\end{array}$ & $\begin{array}{l}N, C \\
N(C)\end{array}$ & $\begin{array}{l}\text { type } \mathrm{C} \\
\text { type } \mathrm{C}\end{array}$ & - \\
\hline $\mathrm{C} \mathrm{II}$ & Freire (1979) & CLAN & - & type A res.lines \\
\hline N I & *Hubený (1981) & $\mathrm{N}$ & type C & - \\
\hline OI & Baschek et al. (1977) & NLWA & - & type A res. lines \\
\hline $\begin{array}{l}\mathrm{Mg} \mathrm{I}, \mathrm{II} \\
\mathrm{Mg} \text { II }\end{array}$ & $\begin{array}{l}\text { Borsenberget et al (1984) } \\
\text { Snijders and Lamers (1975) } \\
\text { Freire Ferrero et al. (1983) }\end{array}$ & $\begin{array}{l}\text { DW } \\
\text { NLW } \\
\text { CLAN }\end{array}$ & type $\mathrm{C}$ & type A res. lines \\
\hline Si I & $\begin{array}{l}\text { Leckrone and Snijders (1979) } \\
\text { Vauclair et al. (1979) }\end{array}$ & $\begin{array}{l}N \\
D\end{array}$ & $\begin{array}{l}\text { type C } \\
\text { type C }\end{array}$ & - \\
\hline Si II & Freire (1979) & CLAN & - & type A res.lines \\
\hline SI & *Hubený (1981) & $\mathbf{N}$ & type C & - \\
\hline $\mathrm{Ca}$ II & $\begin{array}{l}\text { Snijders (1975) } \\
\text { Freire et al. (1978) } \\
\text { Borsenberger et al. (1981) }\end{array}$ & $\begin{array}{l}\text { N } \\
\text { CLAN } \\
\text { DWLN }\end{array}$ & type $\mathrm{C}$ & $\begin{array}{l}\text { type A res.lines } \\
\text { (competition } \\
\text { with } \mathrm{C} \text { ) }\end{array}$ \\
\hline Mn III & Alecian and Michaud (1981) & $\mathrm{D}$ & - & - \\
\hline Sr II & $\begin{array}{l}\text { Praderie (1976) } \\
\text { Borsenberger et al. (1981) }\end{array}$ & $\begin{array}{l}\text { NLW(D) } \\
\text { DWN }\end{array}$ & $\begin{array}{l}\text { type } \mathrm{C} \\
\text { type } \mathrm{C}\end{array}$ & $\begin{array}{l}\text { weak type A } \\
\text { (competition } \\
\text { with C) }\end{array}$ \\
\hline $\mathrm{Ba}$ II & Borsenberger et al. (1984) & DW & type C & - \\
\hline \multicolumn{5}{|c|}{$\begin{array}{l}\text { Purpose: } \\
\text { D - diffusion theory calculations } \\
\text { N - discussion of NLTE effects } \\
\text { A - abundance determination for individual stars } \\
\text { L - detailed line profile calculation } \\
\text { W - calculation of equivalent widths }\end{array}$} \\
\hline
\end{tabular}


C - search for chromospheres

Asterisk $\left(^{*}\right)$ denotes calculations performed self -consistently with the global atmospheric structure

\subsection{Abundance Determinations}

Experience gained in the aforementioned NLTE studies (see also Freire and Praderie 1974; Dumont et al. 1975) enables some conclusions to be drawn concerning the reliability of the various methods of abundance determinations. We shall try to summarize the main advantages and drawbacks of the individual methods in Table 3.

TABLE 3. Comparison of Various Methods of Abundance Determinations.

\begin{tabular}{|c|c|c|c|}
\hline Means & Advantages & Drawbacks & Problems \\
\hline $\begin{array}{l}\text { Strong } \\
\text { resonance } \\
\text { lines } \\
\text { (profiles) }\end{array}$ & $\begin{array}{l}\text {-little influenced by } \\
\text { blends } \\
\text {-simple atomic } \\
\text { models sufficient } \\
\text {-smaller uncertainties } \\
\text { in atomic parameters }\end{array}$ & $\begin{array}{l}\text { NLTE obligatory } \\
\text { (but) }\end{array}$ & $\begin{array}{l}\text { - partial redis- } \\
\text { tribution } \\
\text { (interstellar } \\
\text { absorption) }\end{array}$ \\
\hline $\begin{array}{l}\text { Intermediate } \\
\text { lines } \\
\text { (profiles } \\
\text { eq. widths }\end{array}$ & $\begin{array}{l}\text {-LTE source function } \\
\text { probably satisfactory } \\
\text { (but ionization } \\
\text { shifts) }\end{array}$ & $\begin{array}{l}\text {-uncertainty in } \\
\text { using LTE } \\
\text {-difficult NLTE } \\
\text { (many levels) } \\
\text {-influenced by } \\
\text { blends! }\end{array}$ & $\begin{array}{l}\text {-atomic data! } \\
\text {-Zeeman broaden- } \\
\text { ing }\end{array}$ \\
\hline $\begin{array}{l}\text { Weak lines } \\
\text { (eq. widths) }\end{array}$ & $\begin{array}{l}\text {-LTE source function } \\
\text { probably satisfactory } \\
\text { (but ionization } \\
\text { shifts) }\end{array}$ & $\begin{array}{l}\text {-influenced by } \\
\text { blends! }\end{array}$ & $\begin{array}{l}\text {-atomic data } \\
\text {-Zeeman broaden- } \\
\text { ing }\end{array}$ \\
\hline
\end{tabular}

It appears that the most advantageous method for accurate abundance determinations is the method of detailed comparison of the observed and predicted profiles of resonance lines (supplemented, if possible, by an analogous comparison for the lowest subordinate lines). It is, however, important to calculate the theoretical profiles in NLTE and to account, at least approximately, for the partial redistribution effects (cf. Hubeny 1985, and references therein). As compared to classical methods, the present one requires more complicated calculations, yet this drawback is outweighed by an increased accuracy of the deduced abundances, which may easily be pushed up to $0.05-0.1$ dex. 


\section{LINE BLANKETING}

Excellent reviews of various aspects of line blanketing have been presented by Gustafsson (1981) and Carbon $(1979,1984)$. In this paper we avoid all the numerical aspects and restrict ourselves to the three following questions which appear to be the most important from the point of view of the spectroscopic diagnostics of CP stars:

1) Why is the LTE treatment of line blanketing so successful in explaining observations (i.e. both in construction model atmospheres and calculating detailed synthetic spectra)?

2) Yet, why and when is an NLTE treatment necessary and better?

3) What are the prospects of calculating blanketed model atmospheres and detailed synthetic spectra in an NLTE treatment?

We shall discuss these questions in turn.

5.1 Why is LTE Of ten Satisfactory?

The opacity in a given line $\mathrm{i} \rightarrow \mathrm{j}$ may be expressed (neglecting stimulated emission for convenience) as

$$
\begin{aligned}
\mathrm{k}_{l}(v) & \left.=\left(\pi \mathrm{e}^{2} / \mathrm{mc}\right) \mathrm{f}_{\mathrm{ij}} \mathrm{b}_{\mathrm{i}} \mathrm{n}_{\mathrm{i}} \mathrm{H}\left[\mathrm{a}, v-\nu_{\mathrm{ij}}^{\mathrm{o}}\right) / \Delta v_{\mathrm{D}}\right] /\left(\sqrt{\pi} \Delta v{ }_{\mathrm{D}}\right) \\
& =\mathrm{k}_{l}(v) \mathrm{b}_{\mathrm{i}}
\end{aligned}
$$

where $\mathrm{k}_{l}$, denotes the LTE opacity, $\mathrm{b}_{\mathrm{i}}$ is the $\mathrm{b}$-factor for the lower level; the other quantities have their standard meanings (see, e.g. Mihalas 1978). To the same degree of approximation, the line source function is given by

$$
\mathrm{S}_{l}=\left(\mathrm{b}_{\mathrm{j}} / \mathrm{b}_{\mathrm{i}}\right) \mathrm{B}_{v} \text {. }
$$

Now, for many weak lines, $b_{i}=b_{j}$ while $b_{i}$ may generally be different from unity (e.g. $b_{i}<1$ for the ionization shift). Then $\mathrm{S}_{l} \simeq \mathrm{B}$, and $\mathrm{k}_{l} \simeq \mathrm{k}_{l}^{*} \mathrm{~b}_{\mathrm{i}}$, i.e. the NLTE description only involves a single multiplicative factor in the opacity. However, $b_{i}$ of ten deviates from unity by relatively small factors while the uncertainty in $\mathrm{g}_{\mathrm{i}} \mathrm{f}_{\mathrm{ij}}$ (the so-called gf-value), or the abundance adopted, may easily be comparable with or larger than $b_{i}$.

This is not to say that LTE is a perfect description. This simple example indicates that there are other sourced of uncertainties in treating line blanketing which may of ten be more important than NLTE itself. In this respect, an interesting conclusion follows from a study by Burger (1981): in computing a sample of a synthetic spectrum with the best available gf -values, and with all the gf-values set to -0.5 , who found that all the observed gross spectral features were satisfactorily reproduced by both calculations. Since even this dramatic change in the oscillator strengths yields acceptable results, it is clear why NLTE, which would probably induce even smaller effects, can of ten be disregarded. Moreover, many spectral features, particularly in the UV, are seriously influenced by blends which may yield spurious asymmetries, shifts, and even appearances of spectral lines (Kurucz 1974); Hubený et al. 1985). Again, a fully LTE treatment seems to be better suited to such problems than the seemingly more exact NLTE study which neglects blends. 


\subsection{Why NLTE Blanketing:}

The arguments in favor of the NLTE approach are precisely the same as those outlined in Section 3.2. However, very little work has been done so far that would demonstrate these methodological considerations quantitatively.

A preliminary analysis of this problem (Hubeny 1981) suggests that the main error in the LTE blanketed model atmospheres is probably not caused by the LTE treatment of numerous weak lines, but rather by the LTE treatment of strong UV continua (C I, Si I, Etc.). Specifically, LTE largely overestimates the far UV opacity, thereby yielding too high backwarming in the continuum-forming regions.

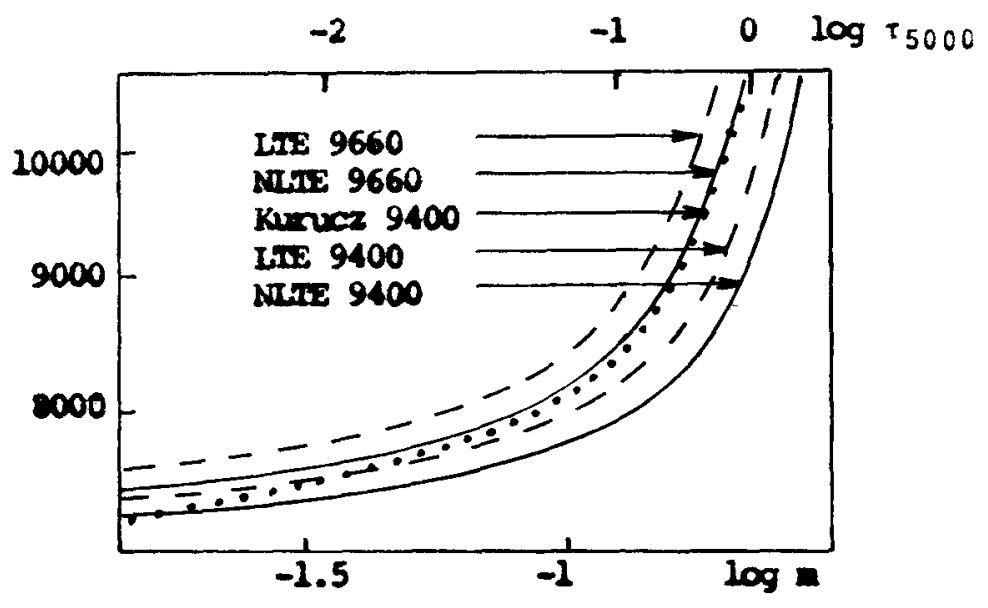

Figure 5. Temperature in the continuum-forming layers for various model atmospheres. Full lines represent NLTE-unblanketed models, broken lines LTEunblanketed models for the indicated values of $\mathrm{T}_{\text {eff. }}$. The dots represent Kurucz's LTE blanketed model for $T_{\text {eff }}=9400 \mathrm{~K}$ (after Hubený 1981).

This is illustrated in Figure 5. The LTE treatment, which ignores metal line blanketing, certainly underestimates the temperature in the continuum-forming regions (compare the curves Kurucz 9400 and the LTE 9400) but simultaneous overestimates the temperature as compared to the NLTE model (compare the curves LTE 9400 and NLTE 9400, or LTE 9660 and NLTE 9660). Yet, importantly enough, the difference T(blank., LTE) - T(unblank., LTE) is almost as large as the difference T(unblank., LTE) - T(unblank., NLTE) ! Since an analogous overestimate of the far UV opacity sources is necessarily present in Kurucz's models, the temperature in his models is probably systematically too high.

However, the above considerations do not take into account the influence of metal line blanketing on the ionization rates of carbon, etc., which would somewhat decrease the magnitude of the NLTE effects. Anyway, we may conclude that the effective temperature, deduced by means of Kurucz's models is uncertain by about $200 \mathrm{~K}$ for early A-type stars. Studies (of normal stars), that do not require a higher precision, may employ the Kurucz grid, but studies that aim at a more precise determination of $T_{\text {eff }}$ should wait until NLTE line blanketed models become available.

On the other hand, an almost identical behavior of temperature with depth for the LTE 
line blanketed model with $T_{\text {eff }}=9400$ and the NLTE-unblanketed model with $T_{\text {eff }}=9660$ (see Fig. 5) explains why detailed spectra, computed for both models, will nearly coincide under the same assumptions about line formation. Thus, an LTE spectrum synthesis using Kurucz's models may be quite reliable, provided that LTE is a good approximation for the formation of weak lines, even if the models themselves might be in error; the same applies to the NLTE-unblanketed models. In other words, this illustrates that it is, to a large extent, permissible to consider separately the effects of metal line blanketing on the atmospheric structure and on the synthetic spectra.

The problem of the uppermost layers, where NLTE is obligatory, has barely been tackled seriously so far. It is clear that the two of ten quoted results-large surface cooling in the LTE line blanketed models, and a temperature rise in the NLTE unblanketed models-represent crude oversimplifications of the problem.

\subsection{Perspectives of NLTE Line Blanketing}

The considerations presented above, together with other investigations (Phillips 1977; Anderson 1985), indicate that the problem of construction "reasonable" LTE line blanketed models is not hopeless. To treat millions of spectral lines in NLTE is certainly disastrous. However, it should be kept in mind that a large part of the total effect is due to a relatively small number of lines and continua. It is essential to treat some important lines and continua in NLTE; other lines may be treated in LTE or some approximate NLTE approach (as, for example, with the estimate of ionization shifts for relevant ions). This is perhaps more vital for the future research into the field of line blanketing than adding more and more lines in strict LTE.

The rationale for such an approach stems from the experience gained from numerical calculations: errors in the computed atmospheric structure due to an insufficient treatment of crucial opacity sources (e.g., C I ground-configuration continua in the case of early A stars Hubený 1981; or C IV resonance lines in the case of O stars - Anderson 1985) are equivalent to, or may easily outweigh the combined effect of thousands and thousands of weak lines.

The most promising way forward seems to be a suitable extension of the approach developed by Anderson (1985). He uses a multif requency/multigray algorithm which admits the inclusion of up to about 300 lines and any number of continua in full statistical equilibrium. Cleverly constructed artificial model atoms can extend the number of lines to 3000 or more, and opacity sampling techniques, similar to those employed in the LTE modeling, may account for still weaker lines. This procedure is thus ideally suited to meet the requirement specified above.

\section{CONCLUSION}

Recent work has amply demonstrated that the chemical peculiarities found in CP stars are not an artifact of an inadequate analysis. From this viewpoint, the NLTE physics is not an essential ingredient of our understanding of the $\mathrm{CP}$ phenomenon. However, whenever one looks at a stellar spectrum and tries to deduce from it the details of the physical state of the stellar atmosphere, NLTE physics becomes essential.

We have tried to demonstrate that, although many spectral features and/or many lowprecision observed data may be satisfactorily explained by means of the traditional LTE modeling, there is still a wide class of observations which would require caref ul NLTE studies. Some work has already been carried out, but much more remains to be done. Although very laborious and computationally demanding, this effort will certainly be rewarding.

Acknowledgement: The author gratefully acknowledges the travel grant provided by the International Astronomical Union. 


\section{REFERENCES}

Alecian, G., and Michaud, G. 1981, Ap. J., 245, 226.

Anderson, L. S. 1985, in Progress in Stellar Spectral Line Formation Theory, ed. J.E. Beckman and L. Crivellari, NATO ASI Series (Dordrecht: D. Reidel) p 225.

Artru, M. C., Jamar, C., Petrini, D., and Praderie, F. 1981, Astron. Ap., 96, 380.

Athay, R. 1972, Radiative Transport in Spectral Lines, (Dordrecht: D. Reidel)

Auer, L. H., and Mihalas, D. 1970, Ap. J., 160, 223.

Baschek, B., Scholz, M., and Sedlmayr, E. 1977, Astron. Ap., 55, 375.

Baschek, B., Heck, A., Jaschek, C., Jaschek, M., Köppen, J., Scholz, M., and Wehrse, R. 1984, Astron. Ap., 131, 378.

Bell, R. A., and Dreiling, L. A. 1981, Ap. J., 248, 1031.

Boesgaard, A. M., Heacox, W. D., Wolff, S. C., Borsenberger, J., and Praderie, F. 1982, Ap. J., 259, 723.

Borsenberger, J. 1979, Thèse de 3ème cycle, Université Paris VII.

Borsenberger, J., and Gros, M. 1978, Astron. Ap. Suppl., 31, 291.

Borsenberger, J., and Jamar, C. 1980, Astron. Ap., 91, 247.

Borsenberger, J., Michaud, G., and Praderie, F. 1979, Astron. Ap., 76, 287.

Borsenberger, J., Michaud, G., and Praderie, F. 1981, Ap. J., 243, 533.

Borsenberger, J., Michaud, G., and Praderie, F. 1984, Astron. Ap., 139, 147.

Bóme-Vitense, E. 1981, Ap. J., 244, 938.

Burger, M. 1981, Astron. Ap., 94, 199.

Buser, R., and Kurucz, R. L. 1978, Astron. Ap., 70, 555

Carbon, D. F. 1979, Ann. Rev. Astron. Ap., 17, 513.

Carbon, D. F. 1984, in Methods in Radiative Transfer, ed. W. Kalkofen, (Cambridge: University Press), p. 395.

Carpenter, K. G. 1985, Ap. J., 289, 660.

Cowley, C. R. 1980, Vistas Astron., 24, 245.

Cowley, C. R. 1981, in Upper Main Sequence Chemically Peculiar Stars, 23rd Liège Astrophys. Coll., Université de Liège, p. 5.

Cowley, C. R., and Adelman, S. J. 1983, Quart. J. Roy. Astron. Soc., 24, 393.

Dreiling, L. A., and Bell, R. A. 1980, Ap. J., 241, 736.

Dumont, S., Heidemann, N., Jefferies, J. T., and Pecker, J.-C. 1975, Astron. Ap., 40, 127.

Frandsen, S. 1974, Astron. Ap., 37, 139.

Freire, R. 1979, Astron. Ap., 78, 148.

Freire, R., and Praderie, F. 1974, Astron. Ap., 37, 117.

Freire, R., Czarny, J., Felenbok, P., and Praderie, F. 1978, Astron. Ap., 68, 89.

Freire Ferrero, R., Gouttebroze, P., and Kondo, Y. 1983, Astron. Ap., 121, 59.

Gustafsson, B. 1981, in Physical Processes in Red Giants, ed. I. Iben, Jr., and A. Renzini (Dordrecht: D. Reidel), p. 25.

Hubený, 1. 1980, Astron. Ap., 86, 225.

Hubený, I. 1981, Astron. Ap., 98, 96.

Hubený, I. 1985, in Progress in Spectral Line Formation Theory, ed. J. E. Beckman and L. Crivellati, NATO ASI Series (Dordrecht, D. Reidel), p. 27.

Hubený, I., Stefl, S., and Harmanec, P. 1985, Bull. Astron. Inst. Czechosl., 36, 214.

Jamar, C. 1980, Astron. Ap., 89, 22.

Jamar, C., Marcau-Hercot, D., and Praderie, F. 1978, Astron. Ap., 63, 155.

Kudritzki, R.-P. 1973, Astron. Ap., 28, 103.

Kurucz, R. L. 1974, Ap. J., 188, L 21.

Kurucz, R. L. 1979, Ap. J. Suppl., 40, 1.

Kurucz, R. L., and Furenlid, I. 1979, Smithsonian Ap. Obs. Spec. Rep. No. 387.

Lane, M. C., and Lester, J. B. 1984, Ap. J., 281, 723.

Leckrone, D. S., Fowler, J. W., and Adelman, S. J. 1974, Astron. Ap., 32, 327. 
Leckrone, D. S., and Snijders, M. A. J. 1979, Ap. J. Suppl., 39, 549.

Linsky, J. L. 1985, in Progress in Spectral Line Formation Theory, ed. J. E. Beckman and L. Crivellari, NATO ASI Series (Dordrecht, D. Reidel), p. 1.

Madej, J. 1983, Acta Astron., 33, 1.

Maitzen, H. M., and Muthsam, H. 1980, Astron. Ap., 83, 334.

Malagnini, M. L., Faraggiana, R., Morossi, C., and Crivellari, L. 1982, Astron. Ap., 114, 170.

Malagnini, M. L., Faraggiana, R., and Morossi, C. 1983, Astron. Ap., 128, 375.

Michaud, G. 1970, Ap. J., 160, 641 .

Michaud, G. 1981, in Upper Main Sequence Chemically Peculiar Stars, 23rd Liège Astrophys. Coll., Université de Liège, p. 355.

Mihalas, D. 1972, Non-LTE Model Atmospheres for $B$ and $O$ stars, NCAR-TN/STR 76.

Mihalas, D. 1978, Stellar Atmospheres, (2nd ed. San Francisco: Freeman).

Muthsam, H. 1978, Astron Ap. Suppl., 35, 107.

Muthsam, H. 1979, Astron. Ap., 73, 159.

Muthsam, H., and Cowley, C. R. 1984, Astron. Ap., 130, 348.

Muthsam, H., and Stepien, K. 1980, Astron. Ap., 86, 240.

Muthsam, H., and Weiss, W. W. 1978, Astron. Ap., 69, 155.

Peterson, D. M. 1970, Ap. J., 161, 685.

Peytremann, E. 1974, Astron. Ap., 33, 203.

Phillips, A. P. 1977, Mon. Not. Roy. Astron. Soc., 181, 777.

Praderie, F. 1976, in Physics of Ap Stars, IAU Coll. 32, ed. W. W. Weiss, H. Jenkner, and H. J. Wood, Vienna: Universitätssternwarte.

Praderie, F. 1981, Astron. Ap., 98, 92.

Praderie, F. 1982, in Ultraviolet Stellar Classification,, ed. A. Heck and B. Battrick, ESA SP182.

Praderie, F., Boesgaard, A. M., Milliard, and Pitois, M. L. 1977, Ap. J., 214, 130.

Praderie, F., Simonneau, E., and Snow, T. P. 1975, Ap. Space Sci., 38, 337.

Relyea, L. J., and Kurucz, R. L. 1978, Ap. J. Suppl, 37, 45.

Snijders, M. A. J. 1975, Bull. Amer. Astron. Soc., 7, 469.

Snijders, M. A. J. 1977a, Astron. Ap., 60, 377.

Snijders, M. A. J. 1977b, Ap. J., 214, L35.

Snijders, M. A. J., and Lamers, H. J. G. L. M. 1975, Astron. Ap., 41, 245.

Stepien, K. 1978, Astron. Ap., 70, 509.

Stepien, K., and Muthsam, H. 1980, Astron. Ap., 92, 171.

Stepien, K., and Muthsam, H. 1981, Astron. Ap., 100, 159.

Strom, S. E., and Strom, K. M. 1969, Ap. J., 155, 17.

Thomas, R. N. 1983, Stellar Atmospheric Structural Patterns, NASA SP-471.

Underhill, A. B., Divan, L., Prevot-Burnichon, L., and Doazan, V. 1979, Mon. Not, Roy. Astron. Soc., 189, 601.

Underhill, A. B., and Doazan, V. 1982, B Stars with and without Emission Lines, NASA SP456.

Vauclair, S., Hardorp, J., and Peterson, D. M. 1979, Ap. J., 227, 526.

Wolff, S. C. 1983, The A Stars: Problems and Perspectives, NASA SP-463. 